\title{
Existence and polynomial growth of periodic solutions to $\mathrm{KdV}$-type equations
}

\author{
Yung-Fu FANG \\ (Received February 16, 2004; Revised September 3, 2004)
}

\begin{abstract}
We establish local and global existence of periodic solutions for KdV type equations, employing Fourier series and a fixed point argument. We also investigate the polynomial growth of the solutions.

Key words: global existence, fixed point argument, periodic solution, KdV type equation, Polynomial growth.
\end{abstract}

\section{Introduction}

In this paper, we study the existence and the polynomial bound of periodic solutions for the nonlinear dispersive equation of the Korteweg-de Vries type:

$$
\left\{\begin{array}{l}
u_{t}+\partial_{x}^{\alpha} u+u^{k} \partial_{x} u=0, \quad(t, x) \in \mathbb{R}^{+} \times \mathbb{T} \\
u(0, x)=\phi(x)
\end{array}\right.
$$

where $\phi$ is a real function, $\alpha$ a real number, $k$ a positive integer and $\partial_{x}^{\alpha}$ the fractional derivative defined by, via Fourier transform,

$$
\widehat{\partial_{x}^{\alpha}}=i|\xi|^{\alpha} \operatorname{sgn} \xi \text {. }
$$

The function $u$ considered here is a real-valued and space-periodic function. The method used here is the fixed point argument applied to the corresponding integral equation

$$
u(t)=W(t) \phi-\int_{0}^{t} W(t-\tau) w(\tau) d \tau,
$$

where $W(t)=e^{-t \partial_{x}^{\alpha}}$ and $w=u^{k} \partial_{x} u$, see [B2] and [FG].

The original $\mathrm{KdV}$ equation,

$$
\partial_{t} u+\partial_{x}^{3} u+u \partial_{x} u=0
$$

2000 Mathematics Subject Classification : 35D05. 
was derived in 1895 by Korteweg and de Vries as an approximate model of shallow water waves, see $[\mathrm{KdV}]$. It also has been derived in plasma physics and in the studies of anharmonic lattices, see [MGKr]. Some generalizations of $\mathrm{KdV}$ equation has been used to describe certain physical problems, e.g. KdV-type equations in certain crystalline lattices, see [ABFS]. In 1975, P. Lax [L] constructed a large class of special solutions of the KdV equation which are periodic in space and almost periodic in time. In 1993, Bourgain [B2] proved existence of periodic solutions for generalized KdV equations,

$$
\partial_{x}^{3} u+u^{k} \partial_{x} u=0 .
$$

In 1995, Bourgain [B3] extended the result of local solutions to more general $\mathrm{KdV}$ equation,

$$
\partial_{t} u+\partial_{x}^{2 j+1} u+F(u, \text { lower order terms })=0 .
$$

On the other hand, some fifth order (even 7th order) KdV-type equations,

$$
\partial_{t} u+u^{p} \partial_{x} u+\partial_{x}^{3} u+\partial_{x}^{5} u=0,
$$

also has been considered, see [K]. In 1996, Bourgain [B4] obtained a polynomial bound of higher Sobolev norm of solutions for generalized KdV equations. In 1997, Staffilani [S] improved the existence result and the polynomial bound of solution for equation (4b). In 2004, Colliander eta [CKSTT] gave multilinear estimates for for periodic case and their applications.

It is well known that the KdV equation and some KdV-type equations possess solitary waves and infinitely many conservation laws, see [L] and [MGKr]. For the equation (1), there are three quantities are conserved, namely,

$$
\left\{\begin{array}{l}
\int_{\mathbb{T}} u(t) d x, \\
\int_{\mathbb{T}} u^{2}(t) d x, \text { and } \\
\int_{\mathbb{T}} \frac{1}{2}\left(\partial_{x}^{(\alpha-1) / 2} u\right)^{2}(t) d x-\int_{\mathbb{T}} \frac{1}{(k+1)(k+2)} u^{k+2}(t) d x .
\end{array}\right.
$$

In the nonperiodic case there have been some good results on questions of existence and regularity, see $[\mathrm{KPV} 1] \sim[\mathrm{KPV} 3]$ and $[\mathrm{BKPSV}]$.

The outline of this paper is that we first show the local existence result 
for the initial value problem (1) with $k=1$. The essence of the proof is an a priori estimate inspired by work of J. Bourgain, see [B2] and [B3]. It can be understood as a multiplier estimate on the set of $\mathbb{R} \times \mathbb{Z}$. However the proof of the estimate presented here is different from those of [B2]. It essentially relies on an idea of Zygmund [Z]. Once the local existence is proved, we invoke a conservation law to get global existence. Next we discuss the existence results for the initial value problem (1), (hereafter we write IVP), with higher order nonlinearity $k \geq 2$. In Section 4 , we will give a straightforward proof of the a priori estimate. Finally we will discuss the polynomial bound for solution of IVP (1). The main results of this paper are the following theorems.

Theorem A Let $\alpha \geq 3$. If the initial data of (1) is in $L^{2}$ for $k=1$ and in $H^{(\alpha-1) / 2}$ (and small) for $k \geq 2$, then the initial value problem of (1) is globally well-posed.

Theorem B Let $\alpha \geq 3$ and $3 / 2 \leq(\alpha-1) / 2<s$. If the initial data is in $H^{s}$ and small, then the global solution $u$ satisfies

$$
\|u(t)\|_{H^{s}} \leq C|t|^{2 s} .
$$

\section{Existence Results}

Throughout this paper we call

$$
A(\xi)=|\xi|^{\alpha} \operatorname{sgn} \xi \quad \text { and } \quad S=|\tau-A(\xi)|+1 ;
$$

denote by

$$
\widetilde{g}(t, \xi)=\frac{1}{2 \pi} \int_{0}^{2 \pi} e^{-i x \xi} g(t, x) d x
$$

and by

$$
\widehat{g}(\tau, \xi)=\int_{R} e^{-i t \tau} \widetilde{g}(t, \xi) d t
$$

the Fourier coefficients and the Fourier transforms with respect to the space variable and to both the space-time variables, respectively. First we state the local existence result for IVP (1) with $k=1$ :

Theorem 1 If $\alpha \geq 3$ and the initial data $\phi \in L^{2}\left(H^{s}, s \geq 0\right)$, then the IVP of (1) is locally well-posed. 
To prove the above theorem, we use a fixed point argument and the following a priori estimate whose proof will be given later.

Theorem 2 If $\alpha \geq 2$, then we have the following estimates

$$
\|f\|_{L^{4}(\mathbb{R} \times \mathbb{T})} \leq C\left\|S^{(1+\alpha) /(4 \alpha)} \widehat{f}\right\|_{L^{2}(\mathbb{R} \times \mathbb{Z})}
$$

and its dual

$$
\left\|\widehat{f} / S^{(1+\alpha) /(4 \alpha)}\right\|_{L^{2}(\mathbb{R} \times \mathbb{Z})} \leq C\|f\|_{L^{4 / 3}(\mathbb{R} \times \mathbb{T})} .
$$

Before proving Theorem 1, we consider the corresponding linear problem:

$$
\left\{\begin{array}{l}
u_{t}+\partial_{x}^{\alpha} u+w=0, \quad(t, x) \in \mathbb{R}^{+} \times S^{1} \\
u(0, x)=\phi(x) .
\end{array}\right.
$$

The periodic solution of (9) can be expressed in integral form as follows.

$$
\begin{aligned}
u(t, x)= & \sum_{\xi} \widehat{\phi}(\xi) e^{i(x \xi+t A)} \\
& +2 i \sum_{\xi} e^{i x \xi} \int \frac{e^{i t \tau}-e^{i t A}}{\tau-A} \widehat{w}(\tau, \xi) d \tau .
\end{aligned}
$$

Call $U(t, x)$ and $V(t, x)$ the linear and nonlinear parts of $u$ respectively,

$$
\left\{\begin{array}{l}
U(t, x)=\sum_{\xi} \widehat{\phi}(\xi) e^{i(x \xi+t A)} \\
V(t, x)=2 i \sum_{\xi} e^{i x \xi} \int \frac{e^{i t \tau}-e^{i t A}}{\tau-A} \widehat{w}(\tau, \xi) d \tau .
\end{array}\right.
$$

We want to study the nonlinear part first. Choose cut-off functions $\widehat{a}$ and $\widehat{b}$ such that $\widehat{a}+\widehat{b}=1$, supp $\widehat{a} \subset[-2 R, 2 R]$ and $\operatorname{supp} \widehat{b} \subset\{x:|x| \geq R\}$. Make a decomposition of $V(t, x)$ in the following way.

$$
V(t, x)=H(t, x)+\Psi_{1}(t, x)+\Psi_{2}(t, x),
$$

where 


$$
\left\{\begin{array}{l}
\widehat{H}(\tau, \xi)=\frac{\widehat{b}(\tau-A)}{\tau-A} \widehat{w}(\tau, \xi), \\
\widehat{\Psi}_{1}(\tau, \xi)=\delta(\tau-A) \int \frac{\widehat{b}(\lambda-A)}{\lambda-A} \widehat{w}(\lambda, \xi) d \lambda, \\
\widehat{\Psi}_{2}(\tau, \xi)=\sum_{k} \delta^{(k)}(\tau-A) \widehat{G}_{k}(\xi), \\
\widehat{G}_{k}(\xi)=\frac{i^{k}(2 R)^{k-1}}{k !} \int\left(\frac{\lambda-A}{2 R}\right)^{k-1} \widehat{a}(\lambda-A) \widehat{w}(\lambda, \xi) d \lambda,
\end{array}\right.
$$

where $\delta(\tau)$ is the delta function and $\delta^{(k)}$ is its $k$-th derivative.

Since the solution does not decay in time, it is necessary to localize it in time. We assume that $\psi$ is a cutoff function supported in a neighborhood of 0 and denote $\psi_{\delta}(t)=\psi(t / \delta)$, where $\delta$ is a small number to be determined later. Let

$$
u_{\delta}(t, x)=\psi_{\delta}(t)\left(\Psi_{1}+\Psi_{2}\right)(t, x)+F(t, x) .
$$

The norm used here is defined by

$$
N(u)=\left\|S^{1 / 2} \widehat{u}\right\|_{L^{2}(\mathbb{R} \times \mathbb{Z})} .
$$

We want to prove the following result first.

Theorem 3 Let $u_{\delta}$ be defined as in (14), we have the estimate

$$
N\left(u_{\delta}\right) \leq C\left\|\frac{\widehat{w}}{S^{1 / 2}}\right\|_{L^{2}(\mathbb{R} \times \mathbb{Z})}+C\left\{\sum_{\xi}\left|\int \frac{|\widehat{w}|}{S} d \lambda\right|^{2}\right\}^{1 / 2} .
$$

Proof. For the term $H$, since $S^{2}|\widehat{b}(\tau-A)|^{2} /(\tau-A)^{2} \leq 1$, we get

$$
\left\|S^{1 / 2} \widehat{H}\right\|_{L^{2}}^{2} \leq C\left\|\frac{\widehat{w}}{S^{1 / 2}}\right\|_{L^{2}}
$$

For the term $\Psi_{1}$, since $\int S\left|\widehat{\psi}_{\delta}(\tau-A)\right|^{2} d \tau \leq C(\psi)$, we have

$$
\left\|S^{1 / 2}\left(\widehat{\psi}_{\delta} * \widehat{\Psi}_{1}\right)\right\|_{L^{2}} \leq C\left\{\sum_{\xi}\left|\int \frac{|\widehat{w}|}{S} d \lambda\right|^{2}\right\}^{1 / 2} .
$$


For the last term, using the facts that

$$
\left\|S^{1 / 2} \widehat{t}^{k} \psi_{\delta}\right\|_{L^{2}} \leq C(\psi)(2 \delta)^{k} \quad \text { and } \quad\left\|\widehat{G}_{k}\right\|_{L^{(Z)}} \leq C \frac{(2 R)^{k}}{k !}\left\|\frac{\widehat{w}}{S^{1 / 2}}\right\|_{L^{2}},
$$

we obtain

$$
\left\|S^{1 / 2}\left(\widehat{\psi}_{\delta} * \widehat{\Psi}_{2}\right)\right\|_{L^{2}} \leq C(\psi) e^{4 R \delta}\left\|\frac{\widehat{w}}{S^{1 / 2}}\right\|_{L^{2}} .
$$

We divide the proof for Theorem 1 into several steps. First we state and prove two lemmas. Notice that now $w=u \partial_{x} u$.

\section{Lemma 4}

$$
\left\|\frac{\widehat{w}}{S^{1 / 2}}\right\|_{L^{2}(\mathbb{R} \times \mathbb{Z})} \leq C \delta^{(\alpha-1) / 8 \alpha} N(u)^{2} .
$$

\section{Lemma 5}

$$
\left\{\sum_{\xi}\left|\int \frac{|\widehat{w}|}{S} d \lambda\right|^{2}\right\}^{1 / 2} \leq C \delta^{(\alpha-1) / 8 \alpha} N(u)^{2} .
$$

Proof of Lemma 4. Observe that $|\widehat{w}(\tau, \xi)|$ is bounded by

$$
|\xi| \sum_{\eta} \int|\widehat{u}(\lambda, \eta)||\widehat{u}(\tau-\lambda, \xi-\eta)| .
$$

To cancel out the factor $|\xi|$, notice that

$$
\begin{aligned}
& |(\tau-A(\xi))-[(\lambda-A(\eta))+(\tau-\lambda-A(\xi-\eta))]| \\
& =|-A(\xi)+A(\eta)+A(\xi-\eta)| \geq C|\xi|^{\alpha-1},
\end{aligned}
$$

provided $\xi \neq 0, \eta \neq 0$ and $\xi \neq \eta$. Also observe that $\widehat{w}(\tau, 0)=0$. Assume the average of $u$ is zero, i.e. $\widehat{u}(\tau, 0)=0$, temporarily so that we have $(21)$. (This assumption will be removed later.) For the sake of convenience, we denote

$$
\left\{\begin{array}{l}
C(\lambda, \eta)=(|\lambda-A(\eta)|+1)^{1 / 2}|\widehat{u}(\lambda, \eta)|=S^{1 / 2}|\widehat{u}(\lambda, \eta)| \\
\widehat{F}(\lambda, \eta)=|\widehat{u}(\lambda, \eta)| \text { and } \widehat{G}(\lambda, \eta)=C(\lambda, \eta)=S^{1 / 2}|\widehat{u}(\lambda, \eta)|
\end{array}\right.
$$


Thus we can bound $\widehat{w} / S^{1 / 2}$ by

$$
\begin{aligned}
\int \sum_{\eta} \frac{|\xi| C(\lambda, \eta) C(\tau-\lambda, \xi-\eta)}{(|\tau-A(\xi)|+1)^{1 / 2}(|\lambda-A(\eta)|+1)^{1 / 2}} \\
\times \frac{1}{(|\tau-\lambda-A(\xi-\eta)|+1)^{1 / 2}} d \lambda
\end{aligned}
$$

From (21) one of the following cases happens.

$$
\left\{\begin{array}{l}
|\tau-A(\xi)| \geq \frac{C}{3}|\xi|^{\alpha-1}, \\
|\lambda-A(\eta)| \geq \frac{C}{3}|\xi|^{\alpha-1}, \quad \text { and } \\
|\tau-\lambda-A(\xi-\eta)| \geq \frac{C}{3}|\xi|^{\alpha-1}
\end{array}\right.
$$

For the first case of (24), we have

$$
\begin{aligned}
& \int \sum_{\eta} \frac{C(\lambda, \eta) C(\tau-\lambda, \xi-\eta)}{|\xi|^{(\alpha-3) / 2}(|\lambda-A(\eta)|+1)^{1 / 2}(|\tau-\lambda-A(\xi-\eta)|+1)^{1 / 2}} d \lambda \\
& \leq \widehat{F^{2}}(\tau, \xi) .
\end{aligned}
$$

Taking $L^{2}$ norm on $\widehat{F^{2}}$ and applying Theorem 2, we get

$$
\left\|\widehat{F^{2}}\right\|_{L^{2}} \leq N(u)^{(\alpha+1) / \alpha}\|u\|_{L^{2}}^{(\alpha-1) / \alpha} .
$$

Assume that $u$ is supported by $[-\delta, \delta] \times T$, since $(\alpha+1) /(4 \alpha)<1 / 2$, we have

$$
\|u\|_{L^{2}} \leq \delta^{1 / 4}\|u\|_{L^{4}}
$$

which implies

$$
\|F\|_{L^{4}} \leq C \delta^{(\alpha-1) /(8 \alpha)} N(u) .
$$

For the second case of (24), we have

$$
\begin{aligned}
& \int \sum_{\eta} \frac{C(\lambda, \eta) C(\tau-\lambda, \xi-\eta)}{(|\tau-A(\xi)|+1)^{1 / 2}|\xi|^{(\alpha-3) / 2}\left(|\tau-\lambda-A(\xi-\eta)|+\left.1\right|^{1 / 2}\right.} d \lambda \\
& \leq \frac{\widehat{F G}(\tau, \xi)}{S^{1 / 2}} .
\end{aligned}
$$


Taking $L^{2}$ norm on $\widehat{F G} / S^{1 / 2}$, we have

$$
\left\|\frac{\widehat{F G}(\tau, \xi)}{S^{1 / 2}}\right\|_{L^{2}} \leq C \delta^{(\alpha-1) /(8 \alpha)} N(u)^{2} .
$$

The proof of the last case of (24) is similar to the second one.

Remark To remove the condition that the solution is of zero average, $\widehat{u}(\tau, 0)=0$, we may modify the problem (1) by replacing $\phi$ by $\phi_{1}+\phi_{0}$ and $u$ by $u_{1}+\phi_{0}$, where $\phi_{0}=\int \phi(x) d x=\int u(t, x) d x$. All arguments go through if $A(\xi)$ is replaced by $A(\xi)-\phi_{0} \xi$.

Proof of Lemma 5. Observe that $|\widehat{w}| / S$ is bounded by

$$
\begin{aligned}
\int \sum_{\eta} \frac{|\xi| C(\lambda, \eta) C(\tau-\lambda, \xi-\eta)}{(|\tau-A(\xi)|+1)(|\lambda-A(\eta)|+1)^{1 / 2}} \\
\quad \times \frac{1}{(|\tau-\lambda-A(\xi-\eta)|+1)^{1 / 2}} d \lambda
\end{aligned}
$$

We use the notations denoted in the previous Lemma and distinguish again the cases in (24).

For the first case of (24), we have

$$
\left\{\sum_{\xi}\left(\int \frac{|\widehat{w}(\tau, \xi)|}{|\tau-A|+1} d \tau\right)^{2}\right\}^{1 / 2} \sim\left\{\sum_{\xi}\left(\int \frac{|\xi| \widehat{F^{2}}(\tau, \xi)}{|\tau-A|+|\xi|^{\alpha-1}} d \lambda\right)^{2}\right\}^{1 / 2} .
$$

Let $a(\xi)$ be a nonnegative sequence with unit $l^{2}$-norm, i.e. $\sum_{\xi} a^{2}(\xi)=1$. Using the first one in (24) and

$$
\int \frac{\xi^{2}}{\left(|\tau-A|+|\xi|^{\alpha-1}\right)^{2}} d \tau \leq C,
$$

we can estimate

$$
\sum_{\xi} \int \frac{a(\xi)|\xi| \widehat{F^{2}}(\tau, \xi)}{|\tau-A|+|\xi|^{\alpha-1}} d \tau \leq C \delta^{(\alpha-1) /(4 \alpha)} N(u)^{2} .
$$

Use a duality argument, we get (18).

For the second case of (24), we have

$$
\int \sum_{\eta} \frac{C(\lambda, \eta) C(\tau-\lambda, \xi-\eta)}{(|\tau-A(\xi)|+1)|\xi|^{(\alpha-3) / 2}\left(|\tau-\lambda-A(\xi-\eta)|+\left.1\right|^{1 / 2}\right.} d \lambda
$$




$$
\leq \frac{\widehat{F G}(\tau, \xi)}{S}
$$

Taking $l^{2}$ norm on the integral $\int \widehat{F G} / S d \tau$, we get

$$
\left\{\sum_{\xi}\left(\int \frac{\widehat{F G}(\tau, \xi)}{S} d \tau\right)^{2}\right\}^{1 / 2} \leq C \delta^{(\alpha-1) /(8 \alpha)} N(u)^{2} .
$$

The proof of the last case of (24) is again similar to the second one.

Here we come to the stage that we can prove Theorem 1.

Proof of Theorem 1. First we combine the results of Theorem 3 and Lemmas 4 and 5 to get, for the nonlinear part $V(t, x)$ of the solution,

$$
\left\|S^{1 / 2} \widehat{u}_{\delta}\right\|_{L^{2}} \leq C \delta^{(\alpha-1) /(8 \alpha)} N(u)^{2} .
$$

Define the map by

$$
T u(t, x)=\psi_{\delta}(t) U(t, x)+\psi_{\delta}(t) V(t, x) .
$$

Thus the $N$ norm of $T u$ is bounded by

$$
C\left(\|\phi\|_{L^{2}}+\delta^{(\alpha-1) /(8 \alpha)} N(u)^{2}\right) .
$$

By choosing sufficiently large $M$, we have, for suitable $\delta$ and $R$,

$$
N(u) \leq M \Longrightarrow N(T u) \leq M,
$$

provided that $C(\phi)+\delta^{(\alpha-1) /(8 \alpha)} M^{2} \leq M$.

Next we estimate the difference of $T u$ and $T v$ and get

$$
N(T u-T v) \leq C \delta^{(\alpha-1) /(8 \alpha)}(N(u)+N(v)) N(u-v) .
$$

Therefore, again for suitable $\delta$ and $R$, we obtain

$$
N(T u-T v) \leq \frac{1}{2} N(u-v),
$$

provided that $C \delta^{(\alpha-1) /(8 \alpha)}(N(u)+N(v)) \leq 1 / 2$ which can be satisfied by choosing $\delta$ small for given $M$. By Picard's theorem, the map $T$ is a contraction with respect to the norm $N(u)$, hence it has a unique fixed point.

Remarks The nonlinear term can be replaced by $\partial_{x}^{\gamma} u^{2}$, but $1 \leq \gamma \leq(\alpha-1) / 2$. 
To get global existence we need a conservation law, i.e. $\|u(t)\|_{L^{2}}$ is constant for all time $t$. Then we are able to extend the result to global existence.

Theorem 6 Let $\alpha \geq 3$. If the initial data of (1) is in $L^{2}\left(H^{s}, s \geq 0\right)$, then there is a unique periodic solution for the IVP of (1) which exists for all time.

Remarks The method used here can be applied to the following extension of equation (1)

$$
u_{t}+\partial_{x}^{\alpha} u+\partial_{x}^{\beta} u+u \partial_{x} u=0,
$$

where $1<\beta<\alpha$ and $3 \leq \alpha$. (See $[\mathrm{K}]$ for a particular case called the fifth order $\mathrm{KdV}$-type equation.)

\section{Further Results}

In this section, we want to discuss the IVP of (1) for $k \geq 2$. First we consider the case $k=2$, then $k \geq 3$.

$$
\left\{\begin{array}{l}
u_{t}+\partial_{x}^{\alpha} u+u^{2} \partial_{x} u=0, \quad(t, x) \in \mathbb{R}^{+} \times \mathbb{T}, \\
u(0, x)=\phi(x),
\end{array}\right.
$$

where $\alpha \geq 3$.

Theorem 7 For $k=2$, the IVP of (1) is locally well-posed for data in $H^{1}$ $\left(H^{s}, s \geq 1\right)$, and for specified $\int_{T} \phi^{2} d x$.

To prove the Theorem7, we need the followings.

Lemma 8 (Bourgain [B2])

$$
\begin{aligned}
& \left(f^{2}-\int_{\mathbb{T}} f^{2} d x\right) \partial_{x} f= \\
& \quad \frac{1}{3} \sum_{\substack{\xi-\zeta \neq 0 \\
\xi-\eta \neq 0 \\
\eta+\zeta \neq 0}} \xi \widehat{f}(\eta) \widehat{f}(\zeta) \widehat{f}(\xi-\eta-\zeta) e^{i \xi x}-\sum_{\xi} \widehat{f}(\xi)^{2} \widehat{f}(-\xi) e^{i \xi x} .
\end{aligned}
$$

To estimate $w$ we introduce the following norm and notation.

$$
|||u|||^{2}=\sum\left(1+|\xi|^{2}\right) \int S|\widehat{u}(\tau, \xi)|^{2} d \tau
$$




$$
\begin{gathered}
+\sum\left(1+|\xi|^{2}\right)\left(\int|\widehat{u}(\tau, \xi)| d \tau\right)^{2} ; \\
S=1+|\tau-B(\xi)|=1+|\tau-A(\xi)+c \xi| .
\end{gathered}
$$

Proposition 9 For $u_{\delta}$, we can estimate it as follows.

$$
\begin{aligned}
\|\left.\left|u_{\delta}\right|\right|^{2} \leq & \sum\left(1+|\xi|^{2}\right) \int \frac{|\widehat{w}(\tau, \xi)|^{2}}{S} d \tau \\
& +\sum\left(1+|\xi|^{2}\right)\left(\int \frac{|\widehat{w}(\tau, \xi)|}{S} d \tau\right)^{2} .
\end{aligned}
$$

This proposition can be proved in a similar manner as that in [B2].

Proof. Due to the conservation law, $\int_{\mathbb{T}} u^{2}(t, x) d x=\int_{\mathbb{T}} \phi^{2}(x) d x$, we denote

$$
c=\int_{\mathbb{T}} \phi^{2}(x) d x
$$

and consider the IVP

$$
\left\{\begin{array}{l}
u_{t}+\partial_{x}^{\alpha} u+c \partial_{x} u=0 \\
u(0, x)=\psi(x)
\end{array}\right.
$$

for which the solution can be written as

$$
u(t, x)=S_{t} \psi(x)=\sum_{\xi} \widehat{\psi}(\xi) e^{i(\xi x+(A-c \xi) t)} .
$$

Consider the integral equation

$$
u(t)=S_{t} \phi+\int_{0}^{t} S(t-\tau) w(\tau) d \tau
$$

where $w=\left[\int_{\mathbb{T}} u^{2} d x-u^{2}\right] \partial_{x} u$, which is equivalent to the IVP

$$
\left\{\begin{array}{l}
u_{t}+\partial_{x}^{\alpha}+c \partial_{x} u=\left(\int_{\mathbb{T}} u^{2} d x-u^{2}\right) \partial_{x} u \\
u(0, x)=S_{0} \phi=\phi
\end{array}\right.
$$

We construct a sequence of functions $\left\{u_{k}\right\}$ by

$$
u_{k+1}=\sum_{\xi} \widehat{\phi}(\xi) e^{i(\xi x+B t)}+\sum_{\xi} e^{i \xi x} \int \widehat{w}_{k}(\xi, \tau) \frac{e^{i t \tau}-e^{i B t}}{\tau-B} d \tau
$$


where $w_{k}=\left[\int_{\mathbb{T}} u_{k}^{2} d x-u_{k}^{2}\right] \partial_{x} u_{k}$, and $B=A-c$. Observe that

$$
\begin{aligned}
& \mid(\tau-B(\xi))-[(\lambda-B(\eta))+(\theta-B(\zeta)) \\
&+(\tau-\lambda-\theta-B(\xi-\eta-\zeta))] \mid \\
&\left.\quad \sim|| \xi\right|^{\alpha}-|\eta|^{\alpha}-|\zeta|^{\alpha}-|\xi-\eta-\zeta|^{\alpha} \mid
\end{aligned}
$$

To find a lower bound of (48), assume that $\eta+\zeta \neq 0, \xi-\eta \neq 0$, and $\xi-\zeta \neq 0$.

Case I: if one or two of $|\xi|,|\eta|,|\zeta|$ are larger than the others, then

$$
(48) \geq(|\eta|+|\zeta|+|\xi-\eta-\zeta|)^{\alpha-1} .
$$

Case II: if $|\eta| \sim|\zeta| \sim|\xi-\eta-\zeta|$,

$$
(48) \geq(|\eta|+|\zeta|+|\xi-\eta-\zeta|)^{\alpha-2} .
$$

Apply Bourgain's lemma and use the notation $\Omega(\xi)=\left\{(\eta, \zeta) \in \mathbb{Z}^{2}: \eta+\right.$ $\zeta \neq 0, \xi-\eta \neq 0, \xi-\zeta \neq 0\}$, we can rewrite

$$
\begin{aligned}
\widehat{w}(\tau, \xi)= & \frac{1}{3} \sum_{\eta, \zeta \in \Omega(\xi)} \xi \int \widehat{u}(\eta, \lambda) \widehat{u}(\zeta, \theta) \widehat{u}(\xi-\eta-\zeta, \tau-\lambda-\theta) d \lambda d \theta \\
& -\xi \int \widehat{u}(\xi, \lambda) \widehat{u}(\xi, \theta) \widehat{u}(-\xi, \tau-\lambda-\theta) d \lambda d \theta
\end{aligned}
$$

Call

$$
\begin{aligned}
& \widehat{w}_{1}(\tau, \xi)=\frac{|\xi|}{3} \sum_{\eta, \zeta \in \Omega(\xi)} \int|\widehat{u}|(\eta, \lambda)|\widehat{u}|(\zeta, \theta)|\widehat{u}|(\xi-\eta-\zeta, \tau-\lambda-\theta) d \lambda d \theta \\
& \widehat{w}_{2}(\tau, \xi)=|\xi| \int|\widehat{u}|(\xi, \lambda)|\widehat{u}|(\xi, \theta)|\widehat{u}|(-\xi, \tau-\lambda-\theta) d \lambda d \theta
\end{aligned}
$$

So it is sufficient to estimate, for $j=1,2$,

$$
\begin{aligned}
& \sum\left(1+|\xi|^{2}\right) \int \frac{\left|\widehat{w}_{j}(\tau, \xi)\right|^{2}}{S} d \tau \text { and } \\
& \qquad\left(1+|\xi|^{2}\right)\left(\int \frac{\left|\widehat{w}_{j}(\tau, \xi)\right|}{S} d \tau\right)^{2},
\end{aligned}
$$

For the Case I, we distinguish four cases, 


$$
\left\{\begin{array}{l}
|\tau-B(\xi)| \geq|\xi|^{\alpha-1} \\
|\lambda-B(\eta)| \geq|\xi|^{\alpha-1} \\
|\theta-B(\zeta)| \geq|\xi|^{\alpha-1} \\
|\tau-\lambda-\theta-B(\xi-\eta-\zeta)| \geq|\xi|^{\alpha-1}
\end{array}\right.
$$

For the Case II, we employ the inequality $(1+|\xi|)|\xi|<C|\eta||\zeta|$. We can control the solution $u$ by the norm ||$\cdot|| \mid$ and get

$$
\left\|\left|T u\left\|\mid \leq C \delta^{(\alpha-1) /(4 \alpha)}\right\|\|u\|^{3} .\right.\right.
$$

Fixed point argument ensures the existence and uniqueness of the solution.

To get global existence we need conservation laws. We first discuss briefly how to derive those conserved quantities given in (5). For the first one, it is straightforward to get that $\int_{\mathbb{T}} u(t) d x=\int_{\mathbb{T}} \phi d x$. The second one can be proved as follows. Multiplying the equation (1) by $u$ and integrating by parts, we get

$$
\int \frac{1}{2} \partial_{t}\left(u^{2}\right) d x+\int u \partial_{x}^{\alpha} u d x=0 .
$$

Using the identity $|\widetilde{u}(t,-\xi)|=|\widetilde{u}(t, \xi)|$, we can prove that the second integral above is 0 which implies that $\|u(t)\|_{L^{2}}$ is conserved. For the last one, we first take the integral operator $\partial_{x}^{-1}$ on the equation, multiply by $u_{t}$ and then integrate by parts.

Next we apply those conservation laws to obtain the boundedness of $H^{(\alpha-1) / 2}$ norm of solution. We first use interpolation inequalities to bound $H^{1}$-norm of $u$, cf. [L]. Let us assume that $u$ is a smooth periodic function temporarily and denote by

$$
\left\{\begin{array}{l}
\int_{\mathbb{T}} u^{2} d x=F_{0}, \quad \max |u(x)|=M, \\
\int_{\mathbb{T}}\left(\partial_{x}^{(\alpha-1) / 2} u\right)^{2}-\frac{2 u^{k+2}}{(k+1)(k+2)} d x=F_{1}, \quad \text { and } \quad \int_{\mathbb{T}} u_{x}^{2} d x=S .
\end{array}\right.
$$

Since $u$ is smooth, there exists a point $x_{0}$ such that

$$
u^{2}\left(x_{0}\right)=\int_{T} u^{2}(x) d x=F_{0},
$$


we apply the identity $u(x)=u\left(x_{0}\right)+\int_{x_{0}}^{x} u_{x} d x$ to get

$$
u^{2}(x) \leq 2 u^{2}\left(x_{0}\right)+2 \int u_{x}^{2} d x \leq 2 F_{0}+2 S .
$$

This implies that $M^{2} \leq 2 F_{0}+2 S$. On the other hand, we can bound $S$ as follows.

$$
\begin{aligned}
S=\int_{\mathbb{T}} u_{x}^{2} d x & \leq C\left(\int_{\mathbb{T}}\left(\partial_{x}^{(\alpha-1) / 2} u\right)^{2} d x+\int_{T} u^{2} d x\right) \\
& \leq C\left(F_{1}+F_{0}+\frac{2 M^{k}}{(k+1)(k+2)} F_{0} d x\right) .
\end{aligned}
$$

Hence we have

$$
M^{2} \leq 2 F_{0}+2 C\left(F_{1}+F_{0}\right)+\frac{2 C F_{0}}{(k+1)(k+2)} M^{k} .
$$

Thus we can deduce that $M$ is bounded by some constant $C=C\left(F_{0}, F_{1}\right)$, provided that $F_{0}$ and $F_{1}$ are small. Also we have

$$
\int_{\mathbb{T}}\left(\partial_{x}^{(\alpha-1) / 2} u\right)^{2} d x \leq F_{1}+\frac{2 C\left(F_{0}, F_{1}\right)^{k}}{(k+1)(k+2)} F_{0} .
$$

Another approach to bound the $H^{(\alpha-1) / 2}$-norm of solution $u$ is that we interpolate between the $L^{2}$ and $H^{(\alpha-1) / 2}$-norms, see [B1]. Using Hölder and Sobolev inequalities, we have

$$
\begin{aligned}
\int_{\mathbb{T}}\left(\partial_{x}^{(\alpha-1) / 2} u\right)^{2} d x & =F_{1}+\int_{\mathbb{T}} \frac{2 u^{k+2}}{(k+1)(k+2)} d x \\
& \leq F_{1}+C\|u\|_{L^{2}}\|u\|_{L^{2(k+1)}}^{k+1} \\
& \leq F_{1}+C\|\phi\|_{L^{2}}\|u\|_{H^{(\alpha-1) / 2}}^{k+1}
\end{aligned}
$$

This implies that if $\|\phi\|_{H^{(\alpha-1) / 2}}$ is small, then we have

$$
\|u(t)\|_{H^{(\alpha-1) / 2}} \leq C \text { for all } t .
$$

Thus we have proved

Theorem 10 For $k=2$, the IVP of (1) is globally well-posed for small data in $H^{(\alpha-1) / 2}\left(H^{S}, s \geq(\alpha-1) / 2\right)$, and for specified $\int_{T} \phi^{2} d x$.

For the case $k \geq 3$, besides ideas in [B2], we use those in [S] as well. 
Definition i) The space $Y^{s, b}, s, b \geq 0$, is the closure of the Schwartz functions $\mathcal{S}(\mathbb{T} \times \mathbb{R})$, with respect to the norm

$$
\|f\|_{Y^{s, b}}=\max _{i=1,2,3} \nu_{i}^{(s, b)}(f),
$$

where

$$
\left\{\begin{array}{l}
\nu_{1}^{(s, b)}(f)^{2}=\sum_{\xi}(1+|\xi|)^{2 s}\left(\int_{\mathbb{R}}|\widehat{f}|(\tau, \xi) d \tau\right)^{2} \\
\nu_{2}^{(s, b)}(f)^{2}=\sum_{\xi}(1+|\xi|)^{2 s} \int_{\mathbb{R}}|\widehat{f}|^{2}(\tau, \xi)(1+|\tau-A(\xi)|)^{2 b} d \tau \\
\nu_{3}^{(s, b)}(f)^{2}=\sum_{\xi}(1+|\xi|)^{2 s-2} \int_{\mathbb{R}}|\widehat{f}|^{2}(\tau, \xi)(1+|\tau-A(\xi)|)^{2 b+1} d \tau .
\end{array}\right.
$$

Denote the space $Y^{s, b}[-\delta, \delta]$ of functions defined on $\mathbb{T} \times[-\delta, \delta]$ with the restriction norm

$$
\|f\|_{Y^{s, b}[-\delta, \delta]}=\inf \|F\|_{Y^{s, b}},
$$

where the infimum is taken over all the extensions $F$ of $f$ on $\mathbb{T} \times \mathbb{R}$.

ii) The space $\bar{Y}^{s, b}, s, b \geq 0$, is the closure of the Schwartz functions $\mathcal{S}(\mathbb{T} \times \mathbb{R})$, with respect to the norm

$$
\|f\|_{\bar{Y}^{s, b}}=\max _{i=1,2,3,4} \mu_{i}^{(s, b)}(f),
$$

where

$$
\left\{\begin{array}{l}
\mu_{1}^{(s, b)}(f)^{2}=\sum_{\xi}(1+|\xi|)^{2 s}\left(\int_{|\tau-A(\xi)>| \xi \mid}|\widehat{f}|(\tau, \xi) d \tau\right)^{2} \\
\mu_{2}^{(s, b)}(f)^{2}=\sum_{\xi}(1+|\xi|)^{2 s} \int_{|\tau-A(\xi)>| \xi \mid}|\widehat{f}|^{2}(1+|\tau-A(\xi)|)^{2 b} d \tau \\
\mu_{3}^{(s, b)}(f)^{2}=\sum_{\xi}(1+|\xi|)^{2 s-2} \int_{\mathbb{R}}|\widehat{f}|^{2}(\tau, \xi)(1+|\tau-A(\xi)|)^{4 b} d \tau \\
\mu_{4}^{(s, b)}(f)=\left\|\partial_{x}^{s} f\right\|_{L_{t}^{\infty} L_{x}^{2} .}
\end{array}\right.
$$

As in i), we have the space $\bar{Y}^{s, b}[-\delta, \delta]$.

iii) Let $f$ and $g$ be functions on $\mathbb{T} \times[-\delta, \delta]$ and $F$ and $G$ be the 
extensions on $\mathbb{T} \times \mathbb{R}$. Denote

$$
\begin{aligned}
& \left\{\begin{array}{l}
\beta_{F}(t)=\int_{\mathbb{T}} F^{k}(t, x) d x \\
\mathcal{F}(F)(\tau, \xi)=\int_{\mathbb{R}} e^{-i t \tau} e^{i \xi \int_{0}^{t} \beta_{F}(\sigma) d \sigma} \widetilde{F}(t, \xi) d t
\end{array}\right. \\
& \left\{\begin{array}{l}
d_{1}^{s}(F, G)^{2}=\sum_{\xi}(1+|\xi|)^{2 s}\left(\int_{\mathbb{R}}|\mathcal{F}(F)-\mathcal{F}(G)|(\tau, \xi) d \tau\right)^{2} \\
d_{2}^{s}(F, G)^{2}=\sum_{\xi}(1+|\xi|)^{2 s} \int_{\mathbb{R}}|\mathcal{F}(F)-\mathcal{F}(G)|^{2}(1+|\tau-A|)^{2 b} d \tau \\
d_{3}^{s}(F, G)^{2}=\sum_{\xi}(1+|\xi|)^{2 s} \int_{\mathbb{R}}|\mathcal{F}(F)-\mathcal{F}(G)|^{2}(1+|\tau-A|)^{4 b} d \tau .
\end{array}\right.
\end{aligned}
$$

Then denote the metric space by $X_{k}^{s, b}[-\delta, \delta]$ with respect to the metric

$$
d_{*}^{s}(F, G)=\inf _{F G}\left\{\sum_{i} d_{i}^{s}(F, G)\right\} .
$$
$[\mathrm{S}]$.

The space $X_{k}^{s, b}[-\delta, \delta]$ is a complete metric space, $s \geq 1 / 2, b \geq 0$, see

Theorem 11 Consider the IVP (1) for $k \geq 3$. If $\phi \in H^{s}, s \geq(\alpha-1) / 2$, then there exists $\delta=\delta\left(\|\phi\|_{H^{(\alpha-1) / 2}}\right)$ and a unique solution $u$ in the space $X_{k}^{s, b}[-\delta, \delta]$ such that

$$
d_{*}^{s}(u, 0) \leq C\|\phi\|_{H^{s}} .
$$

To prove Theorem 11, we consider the associated problem of IVP (1),

$$
\left\{\begin{array}{l}
v_{t}+\partial_{x}^{\alpha} v+\left(v^{k}-\int_{\mathbb{T}} v^{k} d x\right) \partial_{x} v=0, \quad(t, x) \in \mathbb{R}^{+} \times \mathbb{T} \\
v(0, x)=\phi(x),
\end{array}\right.
$$

Consider

$$
\widetilde{u}(t, \xi)=e^{i \xi \int_{0}^{t} \beta_{v}(\sigma) d \sigma} \widetilde{v}(t, \xi) .
$$

The importance of the IVP (70) is that if $v$ is a solution for the problem, then $u$ given by above is a solution for IVP (1). 
Proposition 12 Let $\phi \in H^{s}$ and $s \geq(\alpha-1) / 2$. Then there exists $\delta=$ $\delta\left(\|\phi\|_{H^{(\alpha-1) / 2}}\right)$ such that the problem $(70)$ is well posed in $Y^{s, 1 / 2}[-\delta, \delta]$ and the solution satisfies the bound

$$
\|v\|_{Y^{s, 1 / 2}[-\delta, \delta]} \leq C\|\phi\|_{H^{s}} .
$$

The proof relies on Bourgain's ideas and following lemma.

Lemma 13 (Bourgain, [B2]) If $w \in Y^{s, 1 / 2}, s \geq 1$ and denote

$$
\begin{aligned}
& P(\tau, \xi)=\left[\psi_{\delta}\left(w^{k}-\beta_{w}\right) \partial_{x} w\right]^{\sim}(\tau, \xi) \\
& \left\{\begin{array}{c}
\left(\sum_{\xi} \int_{\mathbb{R}}(1+|\xi|)^{2 s-2}|P(\tau, \xi)|^{2} d \tau\right)^{1 / 2} \\
\leq C \delta^{\epsilon}\|w\|_{Y^{1,1 / 2}}\|w\|_{Y^{s, 1 / 2}}^{k} \\
\left(\sum_{\xi} \int_{\tau-A(\xi) \leq\left(|\xi|^{2}\right) / 200}(1+|\xi|)^{2 s}|P|^{2} d \tau\right)^{1 / 2} \\
\leq C \delta^{\epsilon}\|w\|_{Y^{1,1 / 2}}\|w\|_{Y^{s, 1 / 2}}^{k}
\end{array}\right.
\end{aligned}
$$

for some $\epsilon>0$.

Proof of Proposition 12. Define the map $T$ on $Y^{s, 1 / 2}[-\delta, \delta]$ such that

$$
\begin{aligned}
\widetilde{T(v)}(t, \xi)= & \psi(t) e^{-i t A(\xi)} \widehat{\phi}(\xi) \\
& +\psi_{\delta}(t) \int_{0}^{t} e^{-i(t-s) A(\xi)} \mathcal{F}\left(\left(v^{k}-\beta_{v}\right) \partial_{x} v\right)(s, \xi) d s .
\end{aligned}
$$

We want to show that the map $T$ is a contraction.

As in Theorem 1, we first split $T(v)$ into linear and nonlinear parts and denoted by $U$ and $V$ respectively.

$$
\left\{\begin{array}{l}
\widetilde{U}(t, \xi)=\psi(t) e^{-i t A(\xi)} \widehat{\phi}(\xi) \\
\widetilde{V}(t, \xi)=\psi_{\delta}(t) \int_{0}^{t} e^{-i(t-s) A(\xi)} \mathcal{F}\left(\left(v^{k}-\beta_{v}\right) \partial_{x} v\right)(s, \xi) d s
\end{array}\right.
$$

To estimate $U$, we follow arguments in [KPV1] and [S] obtain, for $j=$ $1,2,3$,

$$
\nu_{j}^{s}(U) \leq C\|\phi\|_{H^{s}} .
$$


To estimate $V$, we follow Bourgain's argument, and use Lemma 13 and

$$
\left\|\partial_{X}^{s} w\right\|_{L_{t}^{\infty} L_{x}^{2}}^{2} \leq \sum_{\xi}\left(\int_{\mathbb{R}}(1+|\xi|)^{s} \mid \widetilde{w}(\tau, \xi) d \tau\right)^{2} .
$$

We have, for $j=1,2,3$,

$$
\nu_{j}^{s}(V)^{2} \leq C \delta^{2 \gamma}\|v\|_{Y^{1,1 / 2}}^{2}\|v\|_{Y^{s, 1 / 2}}^{2 k} .
$$

Hence we obtain

$$
\|T(v)\|_{Y^{s, 1 / 2}[-\delta, \delta]} \leq C\|\phi\|_{H^{s}}+\delta^{\gamma}\|v\|_{Y^{1,1 / 2}[-\delta, \delta]}\|v\|_{Y^{s, 1 / 2}[-\delta, \delta]}^{k} \cdot
$$

Thus if $\delta=\delta\left(\|\phi\|_{H^{1}}\right)$ is small, then, for $R=C\left(\|\phi\|_{H^{s}}\right), T$ is a contraction from a ball $B_{R}$ into itself.

Next we observe that

$$
\begin{aligned}
\mathcal{F}(T u-T v)(t, \xi)= & \psi_{\delta}(t) \int_{0}^{t} e^{-i(t-s) A(\xi)} . \\
& \mathcal{F}\left(\left[(u-v) P_{k-1}(u, v)-\left(\beta_{u}-\beta_{v}\right)\right] \partial_{x} v\right) . \\
& \mathcal{F}\left(\left(u^{k}-\beta_{u}\right) \partial_{x}(u-v)\right) d s
\end{aligned}
$$

which suggest that we can consider the integral equation

$$
\begin{aligned}
\widetilde{w}(t, \xi)= & \psi_{\delta}(t) \int_{0}^{t} e^{-i(t-s) A(\xi)} . \\
& \left.\mathcal{F}\left(\left[w P_{k-1}(u, v)-\theta(s)\right] \partial_{x} v\right) \mathcal{F}\left(u^{k}-\beta_{u}\right) \partial_{x} w\right) d s,
\end{aligned}
$$

where $P_{k-1}(u, v)$ is a polynomial of degree $k-1$ and $\theta(t)=\int_{\mathbb{T}} w P_{k-1}(u, v) d x$. Let $\Phi$ be the operator defined on $Y^{s, 1 / 2}\left[-\delta_{1}, \delta_{1}\right], \delta_{1}<\delta$, by the above integral equation. We can show that there exists $\delta_{1}=\delta_{1}\left(\|u\|_{Y^{s, 1 / 2}},\|v\|_{Y^{s, 1 / 2}}\right)$ such that $\Phi$ is a contraction from a ball $B_{\rho}$ into itself, for arbitrary $\rho$. By uniqueness, we have $u=v$ almost everywhere on $\left[-\delta_{1}, \delta_{1}\right]$. Repeating the argument finite times, we conclude the proof.

The proof of Theorem 11 is basically the same as that given in [S].

Theorem 14 For $k>2$, the IVP of (1) is globally well-posed for data in $H^{(\alpha-1) / 2}\left(H^{s}, s \geq(\alpha-1) / 2\right)$, with sufficiently small $H^{(\alpha-1) / 2}-$ norm.

\section{Proof of A priori Estimate}

In this part, we want to prove Theorem 2. 
Theorem 2 If $\alpha \geq 2$, then we have the following estimates

$$
\left\{\begin{array}{l}
\|f\|_{L^{4}(\mathbb{R} \times \mathbb{T})} \leq C\left\|S^{(1+\alpha) /(4 \alpha)} \widehat{f}\right\|_{L^{2}(\mathbb{R} \times \mathbb{Z})}, \\
\left\|\frac{\widehat{f}}{S^{(1+\alpha) /(4 \alpha)}}\right\|_{L^{2}(\mathbb{R} \times \mathbb{Z})} \leq C\|f\|_{L^{4 / 3}(\mathbb{R} \times \mathbb{T})}
\end{array}\right.
$$

Proof. First we split the function $f$ into positive and negative parts with respect to the dual of space variable and denote

$$
f=f^{+}+f^{-}=\sum_{\xi \geq 0} e^{i x \xi} \widetilde{f}(t, \xi)+\sum_{\xi<0} e^{i x \xi} \widetilde{f}(t, \xi) .
$$

It suffices to prove that $f^{+}$and $f^{-}$both satisfy the estimate. We will only prove the case of $f^{+}$since the proof for $f^{-}$is similar. Hence we replace $f^{+}$ by $f$ and decompose the function in the following way. Choose a smooth function $\widehat{a}$ with support in $\left[2^{-1}, 2\right]$. Let $\widehat{a}_{j}(\tau)=\widehat{a}\left(2^{-j} \tau\right)$ and $\widehat{a}_{0}=1-\sum \widehat{a}_{j}$. Consider

$$
f(t, x)=\sum_{j} f_{j}(t, x), \quad \text { where } \widehat{f}_{j}(\tau, \xi)=\widehat{a_{j}}\left(\tau-|\xi|^{\alpha}\right) \widehat{f}(\tau, \xi)
$$

Thus we have

$$
\|f\|_{L^{4}}^{2} \leq \sum_{j, k}\left\|f_{j} f_{k}\right\|_{L^{2}}
$$

Observe that $\left(f_{j} f_{k}\right)(t, x)$ can be written as

$$
\iint \sum_{\xi_{1} \xi_{2}} e^{i\left(t\left(\tau_{1}+\tau_{2}\right)+x\left(\xi_{1}+\xi_{2}\right)\right)} \widehat{f}_{j}\left(\tau_{1}, \xi_{1}\right) \widehat{f}_{k}\left(\tau_{2}, \xi_{2}\right) d \tau_{1} d \tau_{2}
$$

We choose a change of variables

$$
\begin{cases}\tau=\tau_{1}+\tau_{2}, & \xi=\xi_{1}+\xi_{2} \\ p=p_{1}+p_{2}, & q=p_{2}\end{cases}
$$

where

$$
\left\{\begin{array}{l}
p_{1}=\tau_{1}-\left|\xi_{1}\right|^{\alpha} \in \Delta_{j}=\left[2^{j-1}, 2^{j+1}\right], \\
p_{2}=\tau_{2}-\left|\xi_{2}\right|^{\alpha} \in \Delta_{k}=\left[2^{k-1}, 2^{k+1}\right] .
\end{array}\right.
$$

(Without loss of generality, we assume that $p_{1}$ and $p_{2}$ are both positive. The case of negative $p_{1}$ and $p_{2}$ can be treated in the same manner.) Thus, 
$f_{j} f_{k}$ can be rewritten as follows.

$$
\left(f_{j} f_{k}\right)(t, x)=\int \sum_{\xi} e^{i(t \tau+x \xi)} \widehat{G}_{j k}(\tau, \xi) d \tau,
$$

where

$$
\left\{\begin{array}{l}
\widehat{G}_{j k}(\tau, \xi)=\int_{\Delta_{k}} \sum_{p \in \Lambda_{j}}\left(\widehat{f}_{j} \widehat{f}_{k}\right)(\tau, \xi, q, p) d q \text { and } \\
\Lambda_{j}(\tau, \xi, q)=\left\{p \in \Delta_{j}+q: \xi_{1}, \xi_{2} \in \mathbb{Z}^{+}\right\} .
\end{array}\right.
$$

Applying Plancherel's Theorem, we have

$$
\left\|f_{j} f_{k}\right\|_{L^{2}}=\left\|\widehat{G}_{j k}\right\|_{L^{2}} .
$$

Without loss of generality we may assume that $j>k$. Observe that

$$
\left\|\widehat{G}_{j k}\right\|_{L^{2}}^{2}=\int \sum_{\xi}\left|\int_{\Delta_{k}} \sum_{p \in \Lambda_{j}}\left(\widehat{f}_{j} \widehat{f}_{k}\right)(\tau, \xi, q, p) d q\right|^{2} d \tau .
$$

Claim

$$
\sup _{\tau, \xi, q}\left|\Lambda_{j}\right| \leq C 2^{j / \alpha} .
$$

Assuming the claim, we get

$$
\begin{aligned}
\left\|\widehat{G}_{j k}\right\|_{L^{2}}^{2} \leq \frac{1}{2^{\{(\alpha-1) /(2 \alpha)\}(j-k)}} \cdot \\
\qquad\left\|S^{(1+\alpha) /(4 \alpha)} \widehat{f}_{j}\right\|_{L^{2}}^{2}\left\|S^{(1+\alpha) /(4 \alpha)} \widehat{f}_{k}\right\|_{L^{2}}^{2} .
\end{aligned}
$$

The case of $j<k$ can be treated in a similar fashion. Thus we have

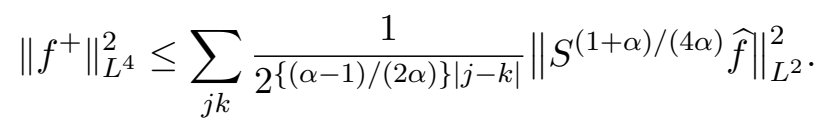

Therefore we obtain

$$
\|f\|_{L^{4}}^{2} \leq\left\|S^{(1+\alpha) /(4 \alpha)} \widehat{f}\right\|_{L^{2}}^{2} \sum_{j k} \frac{1}{2^{\{(\alpha-1) /(2 \alpha)\}|j-k|}}
$$

which implies that $f$ satisfies the estimate. 
Proof of the Claim. Since

$$
\Lambda_{j}(\tau, \xi, q)=\left\{p \in \Delta_{j}+q: \xi_{1}, \xi_{2} \in \mathbb{Z}^{+}\right\},
$$

we can deduce that

$$
\tau-q-2^{j+1} \leq \xi_{1}^{\alpha}+\xi_{2}^{\alpha} \leq \tau-q-2^{j-1} .
$$

Denote

$$
\left\{\begin{array}{l}
A=\tau-q-2^{j+1}, \quad M=3 \cdot 2^{j-1} \\
d(a, b)=|a-b|: \text { the distance between point } a \text { and point } b .
\end{array}\right.
$$

Thus we can rewrite the above inequality as

$$
A \leq \xi_{1}^{\alpha}+\xi_{2}^{\alpha} \leq A+M,
$$

and distinguish the cases, $A \gg M, A \sim M$ and $A \ll M$.

Let $C_{1}$ and $C_{2}$ be the graphs of level curves of $\left|\xi_{1}\right|^{\alpha}+\left|\xi_{2}\right|^{\alpha}$ at $A$ and $A+M$ respectively.

$$
\left\{\begin{array}{l}
C_{1}=\left\{\left(\xi_{1}, \xi_{2}\right):\left|\xi_{1}\right|^{\alpha}+\left|\xi_{2}\right|^{\alpha}=A\right\} \\
C_{2}=\left\{\left(\xi_{1}, \xi_{2}\right):\left|\xi_{1}\right|^{\alpha}+\left|\xi_{2}\right|^{\alpha}=A+M\right\}
\end{array}\right.
$$

Notice that we can only consider the first quadrant. It can be shown easily that, along each level curve, the farthest point to the origin is on the line $\xi_{1}=\xi_{2}$ and the nearest points to the origin are on the axes. Hence $\left|\Lambda_{j}(\tau, \xi, q)\right|$ can be interpreted as the number of lattice points which lie on the straight line $\xi_{1}+\xi_{2}=\xi$ and fall into the region between curves $C_{1}$ and $\mathrm{C}_{2}$.

For the case $A \gg M$, let $C_{3}$ be a circumscribed circle to the curve $C_{2}$,

$$
C_{3}=\left\{\left(\xi_{1}, \xi_{2}\right): \xi_{1}^{2}+\xi_{2}^{2}=2 \sqrt[\alpha]{\frac{(A+M)^{2}}{4}}\right\},
$$

then the largest possible line segment in the region is on the line $l$,

$$
l=\left\{\left(\xi_{1}, \xi_{2}\right): \xi_{1}+\xi_{2}=2 \sqrt[\alpha]{\frac{A}{2}}\right\}
$$

which is tangent to the curve $C_{1}$, see Fig. 1 . Let $a$ and $b$ be the intersections 


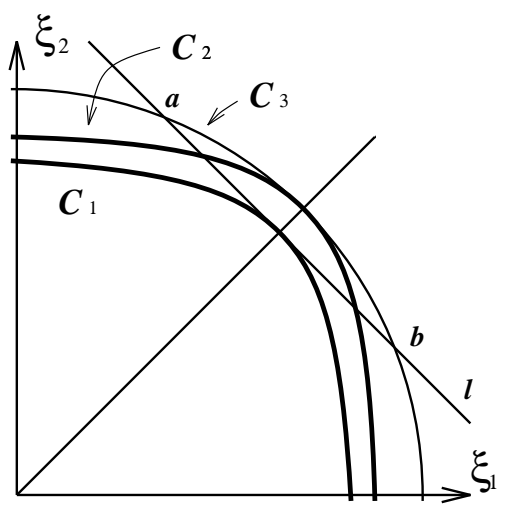

Fig. 1

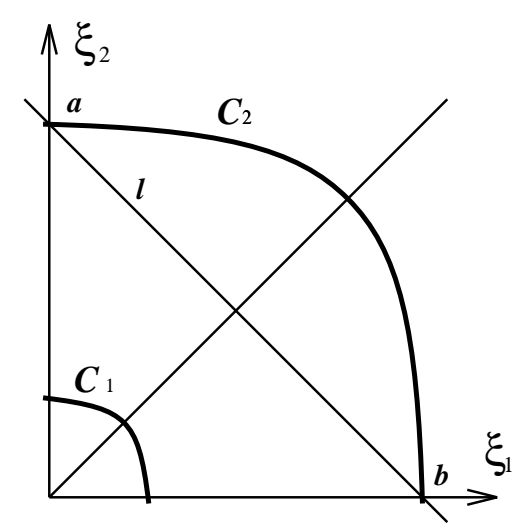

Fig. 2

of the line $l$ and the circle $C_{3}$, then we get

$$
d^{2} \sim \sqrt[\alpha]{\frac{A^{2}}{4}}-\left(2 \sqrt[\alpha]{\frac{A^{2}}{4}}-\sqrt[\alpha]{\frac{(A+M)^{2}}{4}}\right) \leq C \sqrt[\alpha]{M^{2}}
$$

For the case $A \sim M$, the previous argument goes through.

For the case $A \ll M$, since $C_{1}$ is small, we can take the line segment $l$ between two intercepts of $C_{2}$,

$$
l=\left\{\left(\xi_{1}, \xi_{2}\right): \xi_{1}+\xi_{2}=\sqrt[\alpha]{A+M}\right\}
$$

see Fig. 2, and estimate

$$
d \sim \sqrt[\alpha]{A+M} \leq C 2^{j / \alpha} .
$$

Remark It is known that the $L^{6}$-norm estimate is not true. In fact, Bourgain proved the estimate

$$
\left\|\sum_{|n|<N} a_{n} e^{i\left(n x+n^{3} t\right)}\right\|_{6} \ll N^{\epsilon}\left(\sum\left|a_{n}\right|^{2}\right)^{1 / 2}
$$

in his paper [B2]. The optimal estimate should be a $L^{p}$ estimate for $4 \leq$ $p<6$, see [B1] and [FG]. 


\section{The Polynomial Bound}

In the final part, we discuss a polynomial bound for $H^{s}$-norm of the global solution. First we recall two technical lemmas.

Lemma 15 (Kenig-Ponce-Vega, [KPV3]) Assume that $0 \leq \rho \leq 1 / 2$ and $\epsilon>0$ is small. Assume also that $\nu_{2}^{(-\rho+\epsilon, 1 / 2)}(u)$ and $\nu_{2}^{(-\rho+\epsilon, 1 / 2)}(v)$ are bounded and $\int_{\mathbb{T}} u(t, x) d x=\int_{\mathbb{T}} v(t, x) d x=0$. Then

$$
\nu_{2}^{(-\rho-\epsilon,(-1 / 2)+\epsilon)}\left(\partial_{x}(u v)\right) \leq C \nu_{2}^{(-\rho+\epsilon, 1 / 2)}(u) \nu_{2}^{(-\rho+\epsilon, 1 / 2)}(v) .
$$

Lemma 16 (Staffilani, [S]) Assume that $\rho \geq 0, \epsilon>0$ is small and $k \geq 3$. Then

$$
\nu_{2}^{(\rho+\epsilon,(1 / 2)-\epsilon)}\left(\mathcal{X}_{[0,1]} u^{k}\right) \leq C\|u\|_{Y^{1+\rho, 1 / 2}}\|u\|_{Y^{1,1 / 2}}^{k-1} .
$$

Instead of proving Theorem B, we state and prove a more general result.

Theorem 17 Consider IVP (1) and assume that there exists an a-priori bound for the $H^{(\alpha-1) / 2}$-norm of $u$. Then if $\phi \in H^{s}$ and $s \geq(\alpha-1) / 2$, the global solution satisfies the bounds

$$
\begin{cases}\|u(t)\|_{H^{s}} \leq C|t|^{s / \rho} \quad \text { provided } & \rho+1 \leq \frac{\alpha-1}{2}<S \\ \|u(t)\|_{H^{s}} \leq C|t|^{4 s /(2 \rho+(\alpha-3))} & \text { provided } \quad \frac{\alpha-1}{2}<\rho+1<S .\end{cases}
$$

Proof. It is sufficient to show that, for all $t \in[0, \delta / 2]$,

$$
\left\|\partial_{x}^{s} u(t)\right\|_{L_{x}^{2}} \leq\left\|\partial_{x}^{s} \phi\right\|_{L_{x}^{2}}+C\left\|\partial_{x}^{s} \phi\right\|_{L_{x}^{2}}^{1-\delta},
$$

where $\delta^{-1}$ is the exponents in Proposition 9. Since

$$
\begin{aligned}
&\left\|\partial_{x}^{s} u(t)\right\|_{L_{x}^{2}}^{2}=\left\|\partial_{x}^{s} \phi\right\|_{L_{x}^{2}}^{2}+\int_{0}^{t} \frac{d}{d \sigma}\left\|\partial_{x}^{s} u(\sigma)\right\|_{L_{x}^{2}}^{2} d \sigma \\
&=\left\|\partial_{x}^{s} \phi\right\|_{L_{x}^{2}}^{2}-\int_{\mathbb{R}} \int_{\mathbb{T}} x_{[0, t]} u^{k} \partial_{x}\left(\partial_{x}^{s} u\right)^{2} d x d \sigma \\
&+ \text { lower order terms. }
\end{aligned}
$$

Call

$$
J=\int_{\mathbb{R}} \int_{\mathbb{T}} x_{[0, t]} u^{k} \partial_{x}\left(\partial_{x}^{s} u\right)^{2} d x d \sigma
$$


and set

$$
\widetilde{w}(t, \xi)=e^{i \xi \int_{0}^{t} \beta_{u}(\sigma) d \sigma} \widetilde{u}(t, \xi) .
$$

Taking Fourier transform with respect to space variable, multiplying by $e^{i \xi \int_{0}^{t} \beta_{u} d \sigma}$, then taking Fourier transform with respect to time variable, we have

$$
\begin{aligned}
J= & \left.\sum_{\xi} \int_{\mathbb{R}} \widehat{\widehat{x_{[0, t]} w^{k}}}(\tau, \xi) \partial_{x} \widehat{\left(\partial_{x}^{s} w\right)}\right)^{2}(\tau, \xi) d \tau \\
\leq & \sum_{\xi} \int_{\mathbb{R}}\left|\widehat{X_{[0, t]} w^{k}}\right|(\tau, \xi)(1+|\xi|)^{\rho+\epsilon}(1+\mid \tau-A(\xi))^{1 / 2-\epsilon} . \\
& \left|\partial_{x} \widehat{\left(\partial_{x}^{s} w\right)^{2}}\right|(\tau, \xi)(1+|\xi|)^{-\rho-\epsilon}(1+\mid \tau-A(\xi))^{-1 / 2+\epsilon} d \tau \\
\leq & \nu_{2}^{(\rho+\epsilon, 1 / 2-\epsilon)}\left(X_{[0,1]} u^{k}\right) \nu_{2}^{(-\rho-\epsilon,-1 / 2+\epsilon)}\left(\partial_{x}\left(\partial_{x}^{s} u\right)^{2}\right) .
\end{aligned}
$$

Employing Lemmas, Theorem 7, and interpolation between the $H^{(\alpha-1) / 2}$ and the $H^{s}$ norms, we obtain

$$
\begin{aligned}
J & \leq C\|w\|_{Y^{1+\rho, 1 / 2}}\|w\|_{Y^{1,1 / 2}}^{k-1}\left[\nu_{2}^{(-\rho+\epsilon, 1 / 2)}\left(\partial_{x}^{s} w\right)\right]^{2} \\
& \leq C d_{*}^{1+\rho}(u, 0) d_{*}^{1}(u, 0)^{k-1} d_{*}^{s-\rho+\epsilon}(u, 0)^{2} \\
& \leq C\|\phi\|_{H^{1+\rho}}\|\phi\|_{H^{1}}^{k-1}\|\phi\|_{H^{s-\rho+\epsilon}}^{2} .
\end{aligned}
$$

For $\rho+1 \leq(\alpha-1) / 2<S$, interpolation gives

$$
J \leq C\left(\|\phi\|_{H^{(\alpha-1) / 2}}\right)\|\phi\|_{H^{s}}^{2(1-2(\rho-\epsilon) /\{2 s-(\alpha-1)\})} .
$$

If we choose $\epsilon=\rho((\alpha-1) /(2 s))$, we have

$$
\|u(t)\|_{H^{s}} \leq C|t|^{s / \rho} .
$$

For $(\alpha-1) / 2<\rho+1<S$, interpolation gives

$$
J \leq C\left(\|\phi\|_{H^{(\alpha-1) / 2}}\right)\|\phi\|_{H^{s}}^{2(1-\{\rho+(\alpha-3) / 2-2 \epsilon\} /\{2 s-(\alpha-1)\})} .
$$

Choose $\epsilon=\{(\alpha-1)(\rho+(\alpha-3) / 2)\} /\{4 s\}$, we have

$$
\|u(t)\|_{H^{s}} \leq C|t|^{\{4 s\} /\{2 \rho+(\alpha-3)\}} .
$$


Acknowledgment I want to express my gratitude toward Professor M. Grillakis for his inspiring and helpful conversations.

\section{References}

[ABFS] Abdelouhab L., Bona J., Felland M. and Saut J., Nonlocal Models for Nonlinear, Dispersive Waves. Physica D 40 (1989), 360-392.

[B1] Bourgain J., Fourier transform restriction phenomena for certain lattice subsets and applications to nonlinear evolution equations. Part I Schrödinger equations. Geom. Funct. Anal. 3 (1993), 107-155.

[B2] Bourgain J., Fourier transform restriction phenomena for certain lattice subsets and applications to nonlinear evolution equations. Part II The KdV equations. Geom. Funct. Anal. 3 (1993), 209-262.

[B3] Bourgain J., On the Cauchy problem for periodic KdV-type equations. J. Fourier Anal. Appl. (1995), Proceedings of the Conference in Honor of JeanPierre Kahane (Orsay 1993), 17-86 (Special Issue).

[B4] Bourgain J., On the growth in time of higher nnorms of smooth solutions of Hamiltonian PDE. Int. Math. Res. Note 6 (1996), 277-304.

[BKPSV] Birnir B., Kenig C., Ponce G., Svanstedt N. and Vega L., On the Ill-posedness of the IVP for the generalized Korteweg-de Vries and Nonlinear Schrödinger Equations. J. London Math. Soc. (2) 53 (1996), 551-559.

[CGX] Chen Z., Guo B. and Xiang L., Complete Integrability and Analytic solutions of a KdV-type Equation. J. Math. Phys. 31 (1990), 2851-2855.

[CNP] Capel H., Nijhoff F. and Papageorgiou V., Complete Integrability of Lagrangian Mappings and Lattices of KdV type. Phys. Lett. A 155 (1991), 377387.

[CKSTT] Colliander J., Keel M., Staffilani G., Takaoka H. and Tao T., Multilinear estimates for periodic KdV equations and applications. J. Funct. Anal. 211 (2004), 173-218.

[FG] Fang Y.F. and Grillakis M., Existence and Uniqueness for Boussinesq Type Equations on a Circle. Comm. PDE 21 (1996), 1253-1277.

[K] Karpman V., Stationary Solitary Wavesof the Fifth Order KdV-type Equations. Phys. Lett. A 186 (1994), 300-302.

$[\mathrm{KdV}] \quad$ Korteweg D. and de Vries G., On the Change of Form of Long Waves Advancing in a Rectangular Canal, and on a New Type of Long Stationary Waves. Phil. Mag. 39 (1895), 422-443.

[KPV1] Kenig C., Ponce V. and Vega L., The Cauchy Problem for the KdV Equation in Sobolev Spaces of Negative Indices. Duke Math. J. 71 (1993), 1-21.

[KPV2] Kenig C., Ponce V. and Vega L., Higher Order Non-linear Dispersive Equations. Proc. Amer. Math. Soc. 122 (1994), 157-166.

[KPV3] Kenig C., Ponce V. and Vega L., A Bilinear Estimate with Applications to the KdV Equation. J. Amer. Math. Soc. 9 (1996), 573-603. 
[L] Lax P., Periodic Solutions of the KdV Equation. Comm. Pure Appl. Math. 28 (1975), 141-188.

[MGKr] Miura R., Gardner C. and Kruskal M., Korteweg-de Vries Equations and Generalizations, I, II. J. Math. Phys. 9 (1968), 1202-1209.

[S] Staffilani G., On solutions for periodic generalized KdV equations. Internat. Math. Res. Notices (1997), 899-917.

[Z] Zygmund A., On Fourier Coefficients and Transforms of Functions of Two Variables. Studia Math. 50 (1974), 189-201.

Department of Mathematics National Cheng Kung Univ Tainan, Taiwan

fangmath.ncku.edu.tw 